\title{
DA CULTURA DO LITÍGIO PARA ADR: OS VERDADEIROS BASTIDORES DESSA MUDANÇA
}

\section{Fernanda Bragança ${ }^{1}$}

\section{RESUMO}

Esta pesquisa tem o intuito de identificar e analisar os fatores que realmente contribuíram para o desenvolvimento dos métodos alternativos de solução de conflitos nos Estados Unidos a partir de uma revisão bibliográfica norte-americana que faz uma investigação fundamentada deste assunto. Uma vez compreendido este contexto, o estudo analisa como isso se desdobrou na América Latina considerando as peculiaridades locais a partir de artigos e pesquisas específicos que aprofundaram este tema na região, com destaque para o caso brasileiro.

PALAVRAS-CHAVES: meios adequados de solução de conflitos; litígio; judicialização; fatores culturais e políticos

\section{FROM LITIGATION CULTURE TO ADR: THE TRUE BACKGROUND OF THIS CHANGE}

\begin{abstract}
The present research has its focus on identifying and analyzing the factors which actually contribute to the development of alternative dispute resolution in the US, from a bibliographic review where a reasoned investigation on such subject is made. Once understanding this context, the study analyses how it unfolded in Latin America considering the local peculiarities from specific papers and researches that deepen the theme in the region, highlighting the Brazilian case.
\end{abstract}

KEYWORDS: alternative dispute resolution - ADR; litigation; judicialization; cultural and political factors.

\section{INTRODUÇÃO}

Este artigo tem a proposta de investigar o seguinte problema: quais os fatores podem explicar o crescimento do interesse e, consequentemente, do uso de métodos adequados de tratamento de conflitos? De fato, questões institucionais, políticas e culturais permearam toda esta mudança de perspectiva sobre a forma como lidamos com o litígio. O estímulo desse movimento pela resolução alternativa se apresenta enquanto uma grande oportunidade para

\footnotetext{
${ }^{1}$ Doutoranda no programa de Sociologia e Direito da Universidade Federal Fluminense - UFF com séjour doctoral na Université Paris 1 Pantheón-Sorbonne. Mestre e bacharel em Direito pela UFRJ. Pesquisadora do Centro de Inovação, Administração e Pesquisa do Judiciário. Advogada. Mediadora.

Email: nandabra14@gmail.com
} 
analisar quanto uma mudança cultural interage com forças sociais específicas para afetar as disputas.

O objetivo desta pesquisa é apontar e aprofundar sobre os bastidores dessa nova maneira de resolver os conflitos com um paralelo entre as realidades norte-americana e brasileira. Nesse sentido, o estudo foi dividido em três partes: o fomento à $A D R$ no último quarto do século passado nos EUA; os fatores institucionais, políticos e culturais que impulsionaram os meios adequados de solução de conflitos naquele país; e o contexto latinoamericano, com enfoque especial no Brasil.

Esta sistematização do assunto se mostra importante em um contexto em que existem muitos discursos com impressões do senso comum e pouca base científica. A metodologia consistiu em uma revisão bibliográfica criteriosa de doutrina majoritariamente estrangeira que tratou sobre o tema.

\section{O MARCO PÓS O ANO DE 1975 PARA O CRESCIMENTO DO USO DE $A D R$ NOS EUA}

O termo "alternative dispute resolution" tem origem norte-americana e data de 1906, quando foi mencionado pela primeira vez na palestra de Roscoe Pound ${ }^{2}$ em uma conferência da American Bar Association sobre insatisfação com a administração judiciária.

A $A D R$ engloba várias possibilidades na medida em que o termo alternative é demasiadamente genérico e compreende qualquer método alternativo à judicialização, como a negociação, a mediação, arbitragem, a med-arb (combinação de mediação e arbitragem), avaliação neutra precoce, julgamentos sumários do júri, dentre outros.

Neste conjunto, a arbitragem e a mediação são os que mais se destacam e apesar de estarem no mesmo grupo têm características bastante distintas entre si. Enquanto a primeira envolve a decisão de um terceiro, na outra este atua assistindo as partes na negociação e na busca por soluções que lhes convenham. Esses dois métodos foram os que mais encontraram suporte na sociedade e foram regulamentados por diversos países.

\footnotetext{
${ }^{2}$ POUND, Roscoe. The causes of popular dissatisfaction with the administration of Justice. Journal of The American Judicature Society, v. 46, $\mathrm{n}^{\circ}$. 3, pp. 56-71, 1962. Disponível em < https://law.unl.edu/RoscoePound.pdf> acesso em 22 de março de 2020.
} 
A mediação, justamente pela sua ênfase na construção consensual para solução do problema, ganhou a atenção daqueles que valorizam com entusiasmo o comunitarismo, a autonomia do indivíduo e a determinação. Por outro lado, a arbitragem foi mais abraçada pelo setor comercial e supõe custos menores em relação ao Judiciário e tomadores de decisão mais especializados sobre o assunto da demanda.

A visualização do que significou quantitativamente este impulso aos meios adequados de solução de conflitos nos últimos vinte e cinco anos enfrenta barreiras estatísticas. Mesmo nos EUA, um país reconhecido pelo fomento a este tipo de pesquisa, os autores criticam a escassez de dados ${ }^{3}$.

Ainda assim, Richard Chernick ${ }^{4}$ sinaliza em 2004 a prosperidade dessas formas alternativas destacando que os resultados mais expressivos nos EUA ocorreram por conta de iniciativas como: criação de centros comunitários de solução de conflitos; salas de mediação anexas aos tribunais para pequenas disputas cíveis e expansão do uso da arbitragem e avaliação neutra precoce em muitos estados e Cortes federais; regulamentação abundante a nível federal prevendo ADR em tribunais e agências federais; programa de mediação entre pares nas escolas; e rotina de uso da mediação e arbitragem em class actions.

Deborah Hensler ${ }^{5}$ elenca alguns dados em que pese as poucas fontes existentes: a American Arbitration Association aponta um crescimento no número de arbitragens que administrou no período de 1995 a 2002, em que os processos passaram de 62.000 para 230.000. Ainda segundo a autora, mais da metade dos tribunais estaduais e a totalidade das Cortes federais distritais digitais adotaram a mediação para várias categorias de demandas cíveis $^{6}$.

Antes de aprofundar propriamente os fatores que foram decisivos para o crescimento da $A D R$, é preciso destacar que o Judiciário e o Legislativo dos EUA contribuíram com atitudes que tiveram reflexos positivos para o fomento do seu uso. Nesse sentido, a Suprema Corte Americana invalidou restrições previamente confiáveis à arbitragem, invocando os

\footnotetext{
${ }^{3}$ CHASE, Oscar G. ADR and the culture of litigation: the example of the Unites States of America. In: CADIET, Loic; CLAY, Thomas; JEULAND, Emmanuel (Orgs.). Médiation et arbitrage. Alternative Dispute resolution - alternative à la justice ou justice alternatives? Perspectives comparatives. Paris: Lexis Nexis Litec, 2005, p. 136.

${ }^{4}$ Cf. CHERNICK, Richard. ADR comes of age: what can we expect in the future? Pepperdine dispute resolution law journal 4, no. 2, pp. 187-188, 2004.

5 Cf. HENSLER, Deborah R. Our courts, ourselves: how the alternative dispute resolution movement is reshaping our legal system: Penn State Law Review, 108, pp. 165-197, 2003.

${ }^{6}$ Ibid., p. 185.
} 
propósitos pró-arbitragem do Federal Arbitration Act. Este ato foi promulgado em 1925 para fazer valer as sentenças arbitrais nos tribunais federais e, inicialmente, a Suprema Corte teve muita ponderação em enveredar por este método como alternativa ao litígio.

O clima de suspeita em relação ao instituto só apaziguou em 1967, quando a Corte reconheceu a plena aplicação do Federal Arbitration Act - FAA. Em 1983, o tribunal afirmou que qualquer dúvida referente à arbitragem deveria ser resolvida em seu favor quando o problema envolvesse a própria linguagem contratual ou alegação de renúncia, atraso ou defesa à arbitrabilidade ${ }^{7}$.

Em 1984, o tribunal sustentou pela primeira vez que normas estaduais que restringiam a arbitragem de disputas comerciais violavam a $F A A$ e seriam derrubadas ${ }^{8}$. Em 1991, a Suprema Corte adotou uma posição ainda mais surpreendente no caso Gilmer ${ }^{9}$ quando reconheceu a prevalência da cláusula arbitral em um conflito trabalhista mesmo diante das alegações de violação à lei federal antidiscriminação e de um desequilíbrio de poder entre as partes.

O Legislativo, por sua vez, elaborou uma série de atos e estatutos que aceleraram o crescimento da $A D R$ através do encorajamento, financiamento e, às vezes, exigindo a utilização dos mesmos nos tribunais. O Dispute Resolution Act de $1980^{10}$ foi um dos primeiros exemplos desse tipo de legislação que estimulou estados, governos locais e organizações não governamentais a desenvolverem de forma efetiva, justa, barata e rápida mecanismos de solução de conflitos.

Em 1988, o Congresso americano autorizou dez tribunais federais a implementarem programas de arbitragem obrigatórios (mas não vinculativos) para casos menores e mais simples pelo Judicial Improvements and Acess to Justice Act ${ }^{11}$. Em 1990, o Civil Justice Reform $A c t^{12}$ exigiu que todas as cortes distritais criassem planos de redução de custos e do

\footnotetext{
${ }^{7}$ Cf. U.S. SUPREME COURT. Cone Mem. Hosp. vs. Mercury Constr. Corp., 460 U.S. 1 (1983).

Disponível em < https://supreme.justia.com/cases/federal/us/460/1/> acesso em 20 de março de 2020.

${ }^{8}$ Cf. U.S. SUPREME COURT. Southland Corp. v. Keating, 465 U.S. 1 (1984).

Disponível em < https://supreme.justia.com/cases/federal/us/465/1/> acesso em 20 de março de 2020.

${ }^{9}$ Cf. U.S. SUPREME COURT. Gilmer v. Interstate/Johnson Lane Corp., 500 U.S. 20 (1991).

Disponível em < https://supreme.justia.com/cases/federal/us/500/20/> acesso em 20 de março de 2020.

10 GovTrack.us. Dispute Resolution Act, de 12 de fevereiro de 1980. Disponível em < https://www.govtrack.us/congress/bills/96/s423/text> acesso em 20 de março de 2020.

${ }^{11}$ GovTrack.us. Judicial Improvements and Access to Justice Act, de 19 de novembro de 1988. Disponível em < https://www.govtrack.us/congress/bills/100/hr4807/text> acesso em 20 de março de 2020.

12 Congress.Gov. Civil Justice Reform Act. Disponível em <https://www.congress.gov/bill/101stcongress/senate-bill/2027/text> acesso em 20 de março de 2020.
} 
tempo do processo. No mesmo ano, o Administrative Dispute Resolution Act ${ }^{13}$ ordenou o uso de meios adequados de solução de conflitos no contexto das agências administrativas. Com o mesmo intuito, o departamento de justiça também expressou seu encorajamento ao uso de $A D R$ no seio das agências executivas.

Em 1998, o Congresso promulgou o alternative dispute resolution act que determinou que todas as Cortes federais distritais estabelecessem um programa de $A D R$ para disponibilizar aos cidadãos os métodos que entendessem mais apropriados conforme o tipo e a complexidade do litígio. A justificativa dessa legislação apontou que os meios alternativos têm um enorme potencial de prover uma série de benefícios, incluindo a maior satisfação das partes, eficiência no alcance acordos e a diminuição no volume processos pendentes.

\section{OS FATORES CULTURAIS E POLÍTICOS QUE IMPULSIONARAM A $A D R$ NOS DIAS DE HOJE}

Não obstante a importância das medidas adotadas pelo Judiciário e pelo Legislativo norte-americano sobretudo a partir da década de 1980, elas são insuficientes para explicar o crescimento do uso dos meios alternativos nos EUA e em seguida, em todo o mundo.

Uma explicação simples para esta virada após 1975 seria o aumento acentuado de litígios na década anterior que ameaçava sobrecarregar o Judiciário e atrasar a resolução de disputas. Uma pesquisa empírica organizada pelo professor Marc Galater ${ }^{14}$ de fato comprovou o aumento da litigância neste período. Entre 1962 e 1974, o número de ações cíveis nas Cortes distritais federais dobrou de 50.000 para mais de 100.000 .

A partir de uma simples indução econômica parece ser natural que, diante deste quadro, o Poder judicante buscasse outros caminhos para descongestionar e acelerar a finalização dos processos pendentes. E o comportamento das instituições legais relevantes foram mesmo nesta direção.

13 Cf. NATIONAL ARCHIVES. Administrative Dispute Resolution Act (ADRA). Disponível em < https://www.archives.gov/ogis/resources/the-ogis-library/administrative-dispute-resolution-act-adra-s1-p89> acesso em 20 de março de 2020.

${ }^{14}$ Cf. GALANTER, Marc. The Vanishing Trial: an examination of trials and related matters in Federal and State Courts. Journal of Empirical Legal Studies 1, $\mathrm{n}^{\circ}$. 3, pp. 459-570, 2004. Disponível em < http://epstein.wustl.edu/research/courses.judpol.Galanter.pdf> acesso em 20 de março de 2020. 
No entanto, esta simples relação de causa e efeito para rastrear o crescimento da $A D R$ é insuficiente. Richard Posner ${ }^{15}$ mostra que mesmo o período que registrou a maior entrada de casos no Judiciário norte-americano, o impacto na duração do litígio não foi tão significativo. Em 1960, quando se registrou um grande número de ações ajuizadas nos tribunais federais do país, o tempo médio para o julgamento da causa foi de 17,8 meses, e em 1983, chegou a 19 meses onde parece ter se estabelecido. Um abalo real tão leve não seria capaz de provocar uma mudança tão grande na maneira de lidar com as disputas.

Além disso, se a redução dos encargos dos tribunais era o principal motivo dos proponentes de $A D R$, a forma de desvio favorecida tampouco parecia lógica na medida em que consistiu em grande parte no redirecionamento interno através do estabelecimento de arbitragem e mediação anexas à própria Corte de Justiça. E, a menos que esses métodos conseguissem reduzir a quantidade de tempo para alcançar uma solução, eles não serviriam ao objetivo. Assim, era possível que a arbitragem obrigatória, por exemplo, levasse a um pequeno número de prêmios aceitos e a um número maior de novos processos judiciais perante o Judiciário do que justificaria o ônus de imposição geral desta condição de ação ${ }^{16}$.

Em 1975, momento em que as reivindicações em prol do uso de $A D R$ estavam surgindo, não havia qualquer evidência empírica sobre a eficiência dos mesmos em decorrência da sua utilização ainda pouca expressiva. Nos anos seguintes, muitos advogados e juízes creditaram a diminuição do tempo e do custo do processo aos meios adequados de solução de conflitos quando, na verdade, uma série de outras medidas ${ }^{17}$ poderiam ter contribuído para esse fenômeno.

Alguns autores insistiam em afirmar que os tribunais estavam abarrotados de casos e litigantes que, em teoria, não deveriam estar lá. Eles identificavam o problema enquanto uma dependência excessiva da lei para resolver as muitas demandas da sociedade. Eles também apontavam a crescente dependência de instituições legais para resolver disputas como uma ameaça a valores fundamentais, tendo em vista que isso minava as figuras tradicionais de autoridade $^{18}$.

\footnotetext{
${ }^{15}$ POSNER, Richard, A. The Federal Courts: Challenge and Reform. Cambridge: Harvard University Press, 1996, p. 126.

${ }^{16}$ Ibid., pp. 238-239.

${ }^{17}$ Richard Posner enumera outras medidas igualmente relevantes como o aumento do número de magistrados e servidores nos tribunais e a maior carga de trabalho desses profissionais. O Congresso também poderia ter diminuído a competência dos tribunais federais ou redistribuído algumas matérias.

Ibid., pp. 130-131.

${ }^{18}$ Cf. SANDER, FRANK. E. Varieties of dispute processing, 70 F.R.D., 111, 114, 1976.
} 
Esses críticos sinalizavam a necessidade de desvio de algumas disputas para outros fóruns, em particular as que envolvessem pequenas quantias em dinheiro, relações de consumo, bem como outros casos importantes para as pessoas comuns, em oposição aos grandes interesses comerciais ${ }^{19}$. Segundo eles, as Cortes, conforme inicialmente projetadas, não estavam aptas a lidar com esses casos a um custo razoável.

Esta visão foi a que prevaleceu durante uma importante conferência em 1976 que marcou o $70^{\circ}$ aniversário do famoso discurso sobre reforma judicial de Roscoe Pound, reitor da Universidade de Direito de Harvard ${ }^{20}$. O destaque da conferência foram justamente as alternativas à adjudicação tradicional.

Nesta oportunidade, o Presidente da Suprema Corte Americana ressaltou que as reivindicações de baixo valor monetário poderiam ser facilmente solucionadas pelos próprios envolvidos, assim como problemas familiares, guarda de filhos e indenização por lesões resultantes de negligência de médicos e hospitais.

Na mesma linha, Frank Sander afirmou que a sociedade passou a exigir cada vez mais que os tribunais resolvessem disputas que costumavam ser tratadas por outras instituições de sociedade; e esta tendência levaria a um colapso do sistema judiciário ${ }^{21}$. Ele defendeu ainda a criação de uma rede flexível e diversificada de métodos de resolução de disputas (e inclusive combinações entre os mesmos) para atender a tipos específicos de casos.

Em resumo, tanto o posicionamento do Presidente da Suprema Corte quanto o do professor Frank Sanders pareceram mais preocupados em defender os tribunais de um tsunami de demandas mas ao mesmo tempo ressaltaram o potencial da $A D R$ de melhorar as relações comunitárias entre os cidadãos.

O "contra culturalismo" foi um movimento que floresceu na década de 1960 e que exerceu grande influência na resolução de disputas nos EUA, tendo como referências os valores do anti-autoritarismo e da realização da comunidade em que o poder das pessoas era o clamor unificador.

$\mathrm{O}$ entusiasmo em relação à $A D R$ neste contexto de contra culturalismo se deu sobretudo em razão da possibilidade de dispensa da atuação dos advogados e de uma postura mais ativa e decisão dos participantes. Os meios alternativos eram encarados, portanto, como

\footnotetext{
${ }^{19}$ Cf. WARREN, Burger E. Agenda for 2000, A.D. - A need for systematic anticipation, 70 F.R.D. 83, 95, 1976.

${ }^{20}$ AMERICAN ARBITRATION ASSOCIATION. ADR \& the law. New York: JP Juris, 2005, p. 124.

${ }^{21}$ SANDER, FRANK. Ob. Cit., pp. 111-114.
} 
uma oportunidade de escapar da hierarquia judiciária e também do custo de contratação de um profissional do Direito $^{22}$.

Nesse sentido, realça-se o poder construtivo da mediação sobre a informalidade e consensualidade e na possibilidade aberta às partes para que definam seus problemas e objetivos em seus próprios termos a partir de suas premissas individuais: empoderamento e reconhecimento ${ }^{23}$.

Um outro fator que também impactou no crescimento da $A D R$ nos EUA foi o movimento de privatização que marcou o país na década de 1980 com o governo Reagan. Neste período foram realizadas várias mudanças institucionais para reduzir o poder e a presença governamental em alguns setores ao mesmo tempo em que se construiu um aparato de maior autoritarismo em relação à prevenção de crimes, por exemplo ${ }^{24}$. A solução de disputas foi diretamente influenciada por este contexto na medida em que os litígios foram sendo retirados da estrutura estatal.

Em relação aos programas de $A D R$, também é possível dizer que envolveram uma certa privatização, mesmo quando desenvolvidos no âmbito do Judiciário ${ }^{25}$. O árbitro e o mediador são profissionais autônomos (esses profissionais, em sua grande maioria, não compõem a estrutura dos servidores judiciários) e as partes podem estabelecer regras privadas, ou seja, fora do ordenamento jurídico estatal, para reger a situação, desde que respeitem a ordem pública.

Um outro aspecto que alguns autores exploram é o ceticismo em relação às decisões judiciais quanto à uma apuração precisa dos fatos e aplicação correta da lei. Neste cenário, a mediação se torna particularmente atrativa uma vez que os seus resultados não decorrem de uma aplicabilidade estrita da norma jurídica ou da jurisprudência, mas da vontade das partes.

\footnotetext{
${ }^{22}$ RESNIK, Judith. Failing Faith: Adjudicatory Procedure in Decline. The University of Chicago Law Review, $\mathrm{n}^{\circ}$. 53, $\quad$ p. $\quad 537, \quad 1986 . \quad$ Disponível https://chicagounbound.uchicago.edu/cgi/viewcontent.cgi?article=4464\&context=uclrev $>$ acesso em 22 de março de 2020.

23 BUSH, Robert A. Baruch; FOLGER, Joseph P. The promise of mediation: responding to conflict through empowerment and recognition. San Francisco: Jossey-Bass Publishers, 1994, p. 259.

${ }^{24}$ Cf. GARLAND, David. The Culture of control. Chicago: University of Chicago Press, 2001.

${ }^{25}$ Cf. GALANTER, Marc. Ob. Cit.
} 


\section{O CONTEXTO LATINO-AMERICANO}

No final do século XX, a América Latina iniciou seu processo de redemocratização, após anos de experiência com regimes autoritários e economias em recessão. As novas Constituições renovaram a esperança em especial quanto à igualdade perante a lei e à ideia de que todos os habitantes tinham a possibilidade de acessar os sistemas de justiça.

Essas democracias da região falharam no combate à vulnerabilidade que sofria grande parte da população em decorrência da pobreza e da desigualdade. Este fracasso institucional repercutiu sobre o Poder Judiciário que também sofreu com a desconfiança da sociedade, com exceção da Costa Rica, Uruguai e Brasil ${ }^{26}$, países que apresentaram os melhores índices de confiabilidade.

A desregulação e a inserção no comércio internacional marcaram as iniciativas econômicas neste período. Porém, para alcançar um desenvolvimento econômico sustentável, os investimentos exigiam segurança jurídica. Além da preocupação em cumprir os objetivos constitucionais, a demanda por certeza dos agentes econômicos precisava ser atendida; o que configurava mais de um motivo para se preocupar com o acesso ao sistema de justiça ${ }^{27}$.

Nesse sentido, uma série de reformas foram promovidas com a intenção de impulsionar o Judiciário nos moldes em que os Tribunais estavam organizados e, dentre elas, a incorporação de meios adequados de solução de conflitos. Para isso, a região recebeu apoio financeiro da agência americana para desenvolvimento internacional e do Banco Mundial, o qual acompanhou de perto essas reformas na década de 1990.

A principal estratégia desses programas consistiu na promoção da mediação e da arbitragem para o setor privado, em especial, resolver suas disputas. O modelo que serviu de parâmetro para todos os países foi o implementado na Colômbia, onde a Câmara de Comércio de Bogotá liderou a criação de implementação dos centros de mediação e arbitragem. Mesmo

26 Cf. LATINOBARÓMETRO. Opinión Pública LatinoAmericana. Banco de dados. Disponível em < http://www.latinobarometro.org/lat.jsp> acesso em 23 de março de 2020.

${ }^{27}$ Cf. OTEIZA, Eduardo. ADR methods and the diversity of cultures: the latin American case. In: CADIET, Loic; CLAY, Thomas; JEULAND, Emmanuel (Orgs.). Médiation et arbitrage. Alternative Dispute resolution - alternative à la justice ou justice alternatives? Perspectives comparatives. Paris: Lexis Nexis Litec, 2005, pp. 161-177. 
com carências de recursos financeiros, o Estado colombiano conseguiu promover a $A D R$ no seu território e foi tido como exemplo de sucesso a ser seguido por toda a região.

Nos últimos anos, os governos latino-americanos tentaram fortalecer a iniciativa privada por meio de desregulações econômicas e desenvolveram reformas legislativas e políticas públicas para fomentar o uso de $A D R$. O Poder Público investiu fortemente, portanto, em publicidade relacionada às vantagens dos meios alternativos e treinamento de pessoal.

Na América Latina, os meios que mais se destacaram foram a mediação, conciliação e arbitragem, não obstante a ausência de precisão terminológica e de categorização existente entorno deles. No Equador, Costa Rica e Bolívia, por exemplo, os termos mediação e conciliação são empregados como sinônimos indistintamente ${ }^{28}$.

No Brasil, a mediação ganhou destaque a partir da década de 1990 por influência, sobretudo, da lei argentina $n^{\circ} .24 .573$ promulgada em 1995. A primeira iniciativa legislativa brasileira ocorreu somente em 2002, quando a Comissão de Constituição e Justiça aprovou o projeto de lei $n^{0}$. 94. Em sua última versão, este PL propôs a regulamentação da mediação paraprocessual civil que poderia assumir as seguintes feições: prévia, incidental, judicial e extrajudicial $^{29}$.

A doutrina brasileira identifica a presença da arbitragem em nosso sistema jurídico desde a colonização portuguesa ${ }^{30}$. A Constituição de 1985, a primeira Carta Republicana, não tratou expressamente sobre o instituto, mas o mesmo era bastante incentivado. O Texto Constitucional de 1924, em seu art. 160, estabeleceu que as partes podiam nomear juízesárbitros para solucionar litígios cíveis e que suas decisões seriam executadas sem recurso, se as partes, no particular, assim, convencionassem. A Carta de 1934 voltou a aceitar a arbitragem, assegurando à União a competência para legislar sobre o tema.

As Cartas Magnas de 1937, 1946 e de 1967 não fizeram qualquer referência à arbitragem. A Constituição de 1988 tratou sobre o instituto nos artigos $4^{\circ}$, § $9^{\circ}$, VII, e 114 , $\S$

\footnotetext{
${ }^{28}$ Cf. POLANIA, Adriana: ADRs na América Latina. Apresentação na Conferência: Métodos alternativos de solução de controvérsias comerciais: o caminho a seguir para a América Latina e o Caribe. Organizado pelo Fundo Multilateral de Investimentos, Banco Interamericano de Desenvolvimento e Associação Americana de Arbitragem. 26-27 de outubro de 2000, Washington DC, Estados Unidos.

${ }^{29}$ PINHO, Humberto Dalla Bernardina de; CABRAL, Trícia Navarro Xavier. Introdução. In: HALE, Durval; PINHO, Humberto Dalla Bernardina de; CABRAL, Trícia Navarro Xavier. O marco legal da mediação no Brasil. Comentários à lei no. 13.140 de 26 de junho de 2015. São Paulo: Gen, 2016, p. 5.

30 DELGADO, José Augusto. A arbitragem no Brasil: evolução histórica e conceitual. Disponível em <http://www.escolamp.org.br/arquivos/22_05.pdf> acesso em 23 de março de 2020.
} 
$1^{\text {o; }}$ e ainda no seu preâmbulo faz referência à solução pacífica dos conflitos na ordem interna e internacional.

O marco legal atual da arbitragem no Brasil é a Lei $n^{\circ} .9 .307 / 1996$, que esclarece pontos importantes sobre o instituto como por exemplo, quem pode ser árbitro, que a mesma pode ser decidida por equidade ou pelo direito conforme opção das partes, dentre outros. Entretanto, a arbitragem já era utilizada há muitos anos no país sobretudo em conflitos internacionais.

Em que pese o importante papel do Judiciário e do Legislativo brasileiro no sentido de projetar os meios alternativos para a sociedade, em particular a mediação e arbitragem, é preciso enfatizar o forte empenho da iniciativa privada em estimular estes métodos para solucionar os seus conflitos, em particular, os que envolvem um grande investimento financeiro ou infra-estrutura.

Essa discussão é importante e é constantemente debatida. Por exemplo, em evento organizado pela Fundação Getúlio Vargas - FGV em maio de 2018 para tratar sobre os investimentos chineses no Brasil e o papel da arbitragem, mediação e dos meios online de solução de conflitos ficou evidente a preocupação de investidores da China com a complexa ordem jurídica brasileira marcada por um extenso rol normativo, decisões contraditórias e um Judiciário excessivamente moroso. O representante-chefe do China Development bank Corporation no Rio de Janeiro, Song Lei, afirmou nesta oportunidade que recomenda aos empresários de seu país que manifestam interesse em investir aqui, que façam constar nos contratos cláusulas compromissórias escalonadas de mediação e arbitragem (conhecidas também como med/arb) de maneira a evitarem o desgaste e o longo tempo de uma demanda judicial.

O representante da CCIBC citou ainda o caso das refinarias que a Petrobrás comprou na Bolívia; uma situação na qual o Brasil enfrentou sérias dificuldades e estava na posição de investidor. Em $2006^{31}$, o presidente Evo Morales decidiu nacionalizar por meio de um decreto embasado pela Constituição todas as empresas estrangeiras que operavam petróleo e gás no país. Isso resultou numa significativa perda ${ }^{32}$ para a estatal brasileira.

\footnotetext{
31 Notícia que na ocasião foi veiculada pela Folha de São Paulo: <http://www1.folha.uol.com.br/folha/mundo/ult94u95508.shtml> acesso em 28 de maio de 2018.

32 Esses valores foram apurados pelo Jornal OGlobo: <https://oglobo.globo.com/economia/brasil-boliviafecham-acordo-por-us-112-milhoes-4190499> acesso em 24 de março de 2020.
} 
Este movimento não passou despercebido às multinacionais que empregam seu capital na América Latina. Com o intuito de evitar passar por experiências semelhantes, essas empresas procuraram se proteger com instrumentos jurídicos capazes de resolver os entraves fora da esfera política-judiciária da região. Por isso, as mesmas passaram a prever nos seus contratos cláusulas escalonadas de mediação e arbitragem (med/arb) com foro em outro país ${ }^{33}$ considerado confiável para abrigar as negociações sobre a solução do problema.

Por este quadro é possível perceber que a instabilidade e a insegurança jurídica ainda são questões que abalam a América Latina. Neste contexto, os meios adequados de solução de conflitos se apresentam também como um refúgio ao autoritarismo e à incerteza de um Judiciário parcial a favor do Estado.

\section{CONCLUSÃO}

Este artigo desmitifica, portanto, algumas ideias preconcebidas em relação aos meios adequados de solução e as razões do incentivo aos mesmos. O contexto de surgimento e intensificação do uso de $A D R$ é, de fato, muito mais complexo do que em um primeiro momento se pode imaginar. A alta judicialização e o crescimento das demandas a partir da década de 1970 foi um fenômeno sentido em diversos países, inclusive nos EUA. Em que pese o Brasil ser um dos países mais litigantes do mundo, esta dificuldade foi enfrentada por diversos Estados nos anos pós Segunda Guerra com os novos direitos que passaram a ser contemplados nas Constituições e/ou reconhecidos pelos Tribunais.

\footnotetext{
${ }^{33}$ No caso das refinarias da Petrobrás na Bolívia é preciso ressalvar que havia previsão de cláusula de arbitragem, mas que por questões políticas não foi acionada. Em entrevista ao broadcast do Estadão, o ex ministro da Fazenda Rubens Ricupero afirmou na época que o contrato da Petrobras com a YPFB previa uma cláusula - a de número 17 - que no caso de divergência, os dois países primeiro tentariam resolver amigavelmente por negociação, por discussão, e se isso não for possível, o assunto deveria ser submetido à arbitragem da Associação de Arbitragem de Nova York, utilizando as leis do Estado de Nova York, para evitar conflitos de legislação. O contrato estabelecia especificamente a constituição de um tribunal arbitral que julgaria de uma maneira praticamente inapelável e poderia também aplicar multas em dólares sobre o violador, o qual ficaria também responsável por todas as despesas do processo. Caberia à Petrobrás acionar essa cláusula, tendo em vista que o contrato foi firmado entre ela e a empresa boliviana YPFB.

Esta entrevista foi veiculada no portal do Jornal Estadão na web e encontra-se disponível em $<$ https://economia.estadao.com.br/noticias/geral,para-ricupero-bolivia-fez-expropriacao-dapetrobras,20060505p35078> acesso em 24 de março de 2020.
} 
$\mathrm{O}$ incentivo à $A D R$ era apenas uma dentre tantas possibilidades possíveis e a divulgação das vantagens de economia de tempo e custo se deram, em um primeiro momento, sem base em dados estatísticos. A carência de elementos empíricos torna claro que outros fatores foram mais decisivos para a opção por este caminho nos EUA - e que posteriormente foi seguido por outras nações - como o contra culturalismo e o movimento de privatização; o primeiro impulsionou o empoderamento dos indivíduos e o segundo propulsou a solução dos litígios para fora da estrutura estatal.

O fomento aos meios adequados na América Latina ocorreu em razão, sobretudo, de interesses econômicos. Os investimentos estrangeiros na região precisavam ficar resguardados da instabilidade política e a insegurança jurídica presentes na maior parte dos países do continente e, em consequência disso, a alternativa apoiada foi a resolução dos conflitos fora do Judiciário, que além de lento e custoso ficava também sujeito às pressões dos governantes.

\section{REFERÊNCIAS BIBLIOGRÁFICAS}

AMERICAN ARBITRATION ASSOCIATION. ADR \& the law. New York: JP Juris, 2005.

BUSH, Robert A. Baruch; FOLGER, Joseph P. The promise of mediation: responding to conflict through empowerment and recognition. San Francisco: Jossey-Bass Publishers, 1994.

CHASE, Oscar G. ADR and the culture of litigation: the example of the Unites States of America. In: CADIET, Loic; CLAY, Thomas; JEULAND, Emmanuel (Orgs.). Médiation et arbitrage. Alternative Dispute resolution - alternative à la justice ou justice alternatives? Perspectives comparatives. Paris: Lexis Nexis Litec, 2005, pp. 135-151.

CHERNICK, Richard. ADR comes of age: what can we expect in the future? Pepperdine dispute resolution law journal 4, $\mathrm{n}^{\mathrm{o}}$. 2, pp. 187-188, 2004.

CIMAMONTI, Sylvie; PERRIER, Jean-Baptiste (Dir.). Les enjeux de la déjudiciarisation. Paris: LGDJ, 2019.

Congress.Gov. Civil Justice Reform Act. Disponível em $<$ https://www.congress.gov/bill/101st-congress/senate-bill/2027/text > acesso em 20 de março de 2020.

COSTA E SILVA, Paula. A nova face da justiça: os meios extrajudiciais de resolução de controvérsias. Coimbra: Coimbra Editora, 2009. 
DELGADO, José Augusto. A arbitragem no Brasil: evolução histórica e conceitual. Disponível em <http://www.escolamp.org.br/arquivos/22_05.pdf> acesso em 23 de março de 2020.

GALANTER, Marc. The Vanishing Trial: an examination of trials and related matters in Federal and State Courts. Journal of Empirical Legal Studies 1, $n^{\circ}$. 3, pp. 459-570, 2004. Disponível em < http://epstein.wustl.edu/research/courses.judpol.Galanter.pdf> acesso em 20 de março de 2020.

GARLAND, David. The Culture of control. Chicago: University of Chicago Press, 2001.

GovTrack.us. Dispute Resolution Act, de 12 de fevereiro de 1980. Disponível em < https://www.govtrack.us/congress/bills/96/s423/text> acesso em 20 de março de 2020.

GovTrack.us. Judicial Improvements and Access to Justice Act, de 19 de novembro de 1988. Disponível em < https://www.govtrack.us/congress/bills/100/hr4807/text> acesso em $20 \mathrm{de}$ março de 2020.

HENSLER, Deborah R. Our courts, ourselves: how the alternative dispute resolution movement is reshaping our legal system: Penn State Law Review, 108, pp. 165-197, 2003.

LATINOBARÓMETRO. Opinión Pública LatinoAmericana. Banco de dados. Disponível em < http://www.latinobarometro.org/lat.jsp> acesso em 23 de março de 2020.

LESSA NETO, João Luiz. O novo CPC adotou o modelo multiportas!!! E agora?! Revista de Processo, São Paulo, v. 244, p. 427-441, jun. 2015.

NATIONAL ARCHIVES. Administrative Dispute Resolution Act (ADRA). Disponível em < https://www.archives.gov/ogis/resources/the-ogis-library/administrative-dispute-resolutionact-adra-s1-p89> acesso em 20 de março de 2020.

PINHO, Humberto Dalla Bernardina de; CABRAL, Trícia Navarro Xavier. Introdução. In: HALE, Durval; PINHO, Humberto Dalla Bernardina de; CABRAL, Trícia Navarro Xavier. O marco legal da mediação no Brasil. Comentários à lei no. 13.140 de 26 de junho de 2015. São Paulo: Gen, 2016, pp. 5-8.

POLANIA, Adriana: ADRs na América Latina. Apresentação na Conferência: Métodos alternativos de solução de controvérsias comerciais: o caminho a seguir para a América Latina e o Caribe. Organizado pelo Fundo Multilateral de Investimentos, Banco Interamericano de Desenvolvimento e Associação Americana de Arbitragem. 26-27 de outubro de 2000, Washington DC, Estados Unidos.

POSNER, Richard, A. The Federal Courts: Challenge and Reform. Cambridge: Harvard University Press, 1996. 
POUND, Roscoe. The causes of popular dissatisfaction with the administration of Justice. Journal of The American Judicature Society, v. 46, $\mathrm{n}^{\circ}$. 3, pp. 56-71, 1962. Disponível em < https://law.unl.edu/RoscoePound.pdf> acesso em 22 de março de 2020.

RESNIK, Judith. Failing Faith: Adjudicatory Procedure in Decline. The University of Chicago Law Review, nº. 53, pp. 494-560, 1986.

SANDER, FRANK. E. Varieties of dispute processing, 70 F.R.D., 111, 114, 1976.

SILVA, Sabrina Jiukoski da; SILVA, Rafael Peteffi da; SANTOS, Ricardo Soares Stersi dos. A mediação e a conciliação como instrumentos de acesso à justiça e a sua perspectiva a partir do Código de Processo Civil: o contraponto entre a cultura da sentença e a cultura do consenso. Revista Eletrônica de Direito Processual - REDP. Rio de Janeiro. Ano 14. Volume 21. Número 1. Janeiro a abril de 2020, pp. 392-415. Disponível em < https://www.epublicacoes.uerj.br/index.php/redp/article/view/44635/31780> acesso em 20 de março de 2020.

U.S. SUPREME COURT. Cone Mem. Hosp. vs. Mercury Constr. Corp., 460 U.S. 1 (1983). Disponível em < https://supreme.justia.com/cases/federal/us/460/1/> acesso em 20 de março de 2020.

U.S. SUPREME COURT. Southland Corp. v. Keating, 465 U.S. 1 (1984).

Disponível em < https://supreme.justia.com/cases/federal/us/465/1/> acesso em 20 de março de 2020.

U.S. SUPREME COURT. Gilmer v. Interstate/Johnson Lane Corp., 500 U.S. 20 (1991).

Disponível em < https://supreme.justia.com/cases/federal/us/500/20/> acesso em 20 de março de 2020.

WARREN, Burger E. Agenda for 2000, A.D. - A need for systematic anticipation, 70 F.R.D. 83, 95, 1976. 